\title{
Evaluation of vaginal flora and antibiotic prophylaxis in elective hysterectomy in a rural hospital from India
}

\author{
Kalpana V Mahadik', Ashish Pathak ${ }^{2,3}$, Harshada Shah', Manju Raj Purohit ${ }^{2,5}$ \\ 1. Department of Obstetrics and Gynaecology, RD Gardi Medical College, Ujjain, India
}

2. Division of Global Health, Department of Public Health Sciences, Karolinska Institutet, Stockholm, Sweden

3. Department of Paediatrics, RD Gardi Medical College, Ujjain, India

4. Department of Microbiology, RD Gardi Medical College, Ujjain, India

5. Department of Pathology, RD Gardi Medical College, Ujjain, India

doi: 10.3396/IJIC.v9i3.024.13

\begin{abstract}
The incidence of post-operative complications is $11.5 \%$ in developing countries and surgical site infections complicate nearly $20 \%$ of intra-abdominal procedures. Contamination of operative site with vaginal flora is unavoidable and should be treated prior to hysterectomy. This study was conducted to assess the current status of antibiotic prophylaxis in hysterectomy in a rural setting. The study was conducted at Department of Obstetrics and Gynaecology, RD Gardi Medical College, Ujjain, India. Patients of abdominal and vaginal hysterectomy for benign gynaecological diseases were included. Pre-operative vaginal swab was cultured. Patients with culture negative result received ampicillin prophylaxis while culture positive patients received antibiotic of sensitivity. Patients were observed for various post-operative morbidities. Total 109 patients with age range of 37- 76 years were analyzed in the study. Pre-operative vaginal swab culture showed nonpathogenic organisms in $67 \%$ of cultures while pathogenic bacteria in $33 \%$ of cultures. Culture positive results were more often seen in vaginal hysterectomy than in abdominal hysterectomy $(p=0.003)$. Total $25.6 \%$ (28/109) patients showed post-operative morbidities such as vaginal discharge, wound infection, urinary tract infection, and fever in decreasing order of frequency. Only $11.1 \%(4 / 36)$ of patients infected with pathogenic bacteria and 32.8\% (24/73) of patients with non-pathogenic bacteria had complications. Complications were higher in ampicillin prophylaxis patients than in culture specific prophylaxis $(p=.014)$. This study showed the beneficial effect of pre-operative vaginal swab culture in elective hysterectomy and that a culture sensitive antimicrobial coverage is more effective than ampicillin in reducing post-operative complications.
\end{abstract}

Key Words: Vagina and Microbiology; antibiotic prophylaxis; Surgical porcedures, elective; Hysterectomy; India

\section{Corresponding Author}

Kalpana V Mahadik

Department of Obstetrics and Gynaecology

RD Gardi Medical College, Surarsa, Ujjain, India

Email: kalpanavmahadik@hotmail.com 


\section{Introduction}

Infections and fever are the most common postoperative complications which represent a significant burden in terms of patient morbidity, mortality and cost of hospitalization. The incidence of post-operative infection and fever is $11.5 \%$ in developing countries as compared to $5 \%$ in developed countries. ${ }^{1}$ Surgical site infections complicate up to $20 \%$ of intra abdominal procedures. ${ }^{2}$

The vagina is normally colonised with mixed aerobic and anaerobic bacteria species. ${ }^{3,4,5,6}$ Contamination of some operative sites with endogenous organisms from the vagina is unavoidable. The pre-surgery vaginal predominant organisms can readily be cultured to identify risk for causing pathogenic disease. ${ }^{7}$ The asymptomatic vaginal colonization can be treated prior to hysterectomy to avoid post surgical complications. In treated patients, reported reductions in postoperative infectious complications range from 10 to $75 \%{ }^{8,9}$

As early as 1974 Ledger et al. ${ }^{10}$ developed guidelines for antibiotic prophylaxis in gynaecological surgery advocating laboratory and clinical evidence of effectiveness against contaminating organisms. Some data suggests that nearly $30-50 \%$ of antibiotics used in hospitals are prescribed for surgical prophylaxis and $30-90 \%$ of this prophylaxis is inappropriate. ${ }^{11}$

The present study was conducted in a rural university hospital in Ujjain. Many patients undergo gynaecological procedures in our hospital but, the use of prophylactic antibiotic in hysterectomy is mainly determined by the surgeon on individual basis, rather than following an evidence based guideline. The most commonly used regimen is to give two grams of ampicillin. Though Ujjain is one of the largest districts in central India, until now due to lack of adequate baseline information on the pattern of antibiotics prophylaxis usage from our region no proper guidelines have been developed. This is the first study to assess the current status of peri-operative antibiotic prophylaxis in hysterectomy cases and identification of evidence based corrective measures for use of prophylactic antibiotics in patients undergoing gynaecological surgery.

\section{Methodology}

\section{Setting}

This study was conducted at Department of Obstetrics and Gynaecology, RD Gardi Hospital; a 600 bedded university hospital located in rural area of Ujjain, Central India. The hospital caters patients from a low socioeconomic status, nutritionally poor, low priority and poorly literate groups.

\section{Patient}

All in-patients from May 2010 to Dec 2010 with benign gynaecological diseases were initially enrolled in the study. Detailed demographic information, clinical history, clinical examination findings were recorded in a pre-designed questionnaire. Pre-operative haemogram, blood urea nitrogen and sugar level, HIV test, and chest X-ray were also completed. Patients with less than 18 years of age, systemic or associated medical diseases like anaemia (haemoglobin less than $9.0 \mathrm{gm} / \mathrm{dl})$, tuberculosis, diabetes, liver or renal disease, hypertension, cardiac problem or those on antituberculosis treatment, or receiving corticosteroids, immunosuppressive, anti-retroviral therapy were excluded ( $n=14)$ from the study. Patients allergic to antibiotics, currently using or used antibiotics within the week before surgery were also excluded $(n=2)$ from the study. Hence, total 16 patients were excluded from the study.

Abdominal or vaginal hysterectomy of patients was performed. Patients were included when they were clinically diagnosed of benign gynaecological conditions, having expected length of surgery less than two hours, expected blood loss less than $100 \mathrm{mls}$, elective surgery and per-speculum examination revealed no cervical or vaginal discharge.

\section{Vaginal swab collection and culture}

In lithotomy position, a vaginal swab sample was collected with sterile swab on stick from posterior vaginal fornix and external os of uterine cervix after cleaning the vulva with sterile water. The swab stick was immediately put in culture medium for aerobic and anaerobic culture, labelled, stored at $4-6^{\circ} \mathrm{C}$ and transported to microbiology laboratory within an hour of collection. The culture and antibiotic susceptibility was done with the standard laboratory protocol. 


\section{Antibiotic prophylaxis,}

\section{surgical procedure and follow up}

Patients with culture negative vaginal swab received 4g ampicillin (500 mg intravenously six hourly for two days brand SAM-500, Sarabhai Pharmaceuticals) according to the hospital policy, while culture positive patients received antibiotics depending on the sensitivity results (cefoxitin, ciprofloxacin, gentamycin, metronidazole) in standard doses for 5 days. First dose of antibiotics for all patients started half an hour prior to surgery. The abdominal or vaginal hysterectomy was performed using aseptic technique under either general (three patients) or spinal (all other patients) anaesthesia.

Patients were observed for fever and infection during the post operative period. The common causes of complication were urinary tract infection, vaginal cuff infection and surgical site infection. The outcome measures were defined as 1 . Surgical site infection - dressing checked at 48 hours and five days postoperatively for any serosanguinous or purulent discharge, 2. Vaginal cuff infection - serosanguinous or purulent discharge 3 . Fever - temperature $>37^{\circ} \mathrm{C}$ on at least two occasions, 8 hours apart, 4. Urinary tract infection - presence of $>10$ pus cells per high power field of microscopy or culture positive, and 5. Symptomatic upper respiratory tract infection. Patients were discharged from the hospital at the surgeon's discretion after required nursing care. All patients were again followed after six weeks for symptoms and signs of complications.

\section{Data analysis}

Data were analyzed using SPSS Version 13 to assess differences between variables. Chi-square test was done to check the presence of associations. Bivariate logistic regression model analysis was applied to assess the risk factors. P-values $<0.05$ were considered statistically significant.

\section{Ethical Consideration}

Ethical approval was secured from the Institutional ethics committee. A written consent from the medical director and superintendent of the hospital was obtained for conducting the study. An informed written consent was obtained from the patients. All the procedures were conducted according to the clinical and medical need of the patient and not for the sake of study.

Table I. Clinical characteristics of patients in relation to pre-operative vaginal swab culture isolates

\section{Clinical characteristics of patients}

\section{Vaginal swab culture isolates}

Non-pathogenic bacteria Pathogenic bacteria $(\mathrm{N}=36)$

$(\mathrm{N}=73) \mathrm{n}(\%) \quad \mathrm{n}(\%)$

\begin{tabular}{lrr}
\hline $\begin{array}{l}\text { Age (years) } \\
<50\end{array}$ & $33(45.2)$ & $8(22.2)$ \\
$>50$ & $40(54.7)$ & $28(77.7)$ \\
\hline $\begin{array}{l}\text { Parity } \\
<2\end{array}$ & $6(8.2)$ & $1(2.7)$ \\
$>2$ & $67(91.7)$ & $35(97.2)$ \\
\hline $\begin{array}{l}\text { Type of Hysterectomy } \\
\text { Abdominal } \\
\text { Vaginal }\end{array}$ & $40(54.7)$ & $9(25)$ \\
\hline $\begin{array}{l}\text { Length of Surgery } \\
>2 \text { hrs } \\
<2 \text { hrs }\end{array}$ & $33(45.2)$ & $27(75)^{*}$ \\
\hline $\begin{array}{l}\text { Blood loss } \\
>200 m l \\
<200 m l\end{array}$ & $1(1.3)$ & $29(80.5)$ \\
\hline
\end{tabular}

$* p=0.003$ 


\section{Results}

\section{Patient details}

Data from total of 109 patients were analyzed in the study. Patients from all age ranging from 37- 76 years (mean age-58 years) were seen. Patients with preoperative diagnosis of uterine prolapse, uterine fibroid, ovarian mass and dysfunctional uterine bleeding underwent either vaginal or abdominal hysterectomy.

\section{Culture results}

Pre-operative vaginal swab culture showed nonpathogenic organisms in 67\% (73/109) culture while pathogenic bacteria were isolated from 33\% (36/109) cultures. Among pathogenic bacteria Staphylococcus aureus, Escherichia coli, Pseudomonas spp., Klebsiella spp., and Enterococcus spp. and streptococci were isolated in decreasing order of frequency. Patients with pathogenic bacteria were more commonly in those older than 50 years of age. Culture were positive more often in patients who underwent vaginal hysterectomy than abdominal hysterectomy $(p=0.003)$ (table I).

Pathogenic bacteria were isolated on pre-operative vaginal swab culture from most of the uterine prolapse patients while patients with dysfunctional uterine bleeding, uterine fibroid, and ovarian tumour mainly showed non-pathogenic bacteria on culture (table II).

\section{Post-operative morbidities}

A total $25.6 \%$ (28/109) of patients showed different types of post-operative complications. Vaginal discharge, wound infection and urinary tract infection were most common followed by fever etc. The total number and type of complications varied significantly between the patients with nonpathogenic or pathogenic bacteria on vaginal swab culture. Only $11.1 \%$ (4/36) patients infected with pathogenic bacteria and 32.8\% (24/73) patients with non-pathogenic bacteria showed morbidities. Wound infection and urinary tract infection were significantly common in patients with non-pathogenic bacteria on vaginal swab. After controlling for age, parity, and length of surgery using multiple linear regression, the prevalence of complication was statistically significant for blood loss, fibroids, and uterine prolapse.

Table III shows patients receiving ampicillin prophylaxis. Post-operative complicaions was significantly $(p=.014)$ higher where ampicillin

Table II. Vaginal swab culture isolates in various gynaecological diagnosis

Gynaecological diagnosis

Vaginal swab culture isolates

Non-pathogenic bacteria $(n=73) \quad$ Pathogenic

Bacteria $(n=36)$

\begin{tabular}{lrr} 
DUB $(\mathrm{n}=11)$ & $7(9.5)$ & $4(11.1)$ \\
Fibroid $(\mathrm{n}=30)$ & $24(33)$ & $6(16.6)$ \\
Prolapse $(\mathrm{n}=60)$ & $34(46.5)$ & $26(72)$ \\
Ovarian Mass $(\mathrm{n}=8)$ & $8(10.9)$ & 0 \\
\hline
\end{tabular}

DUB = Dysfunctional uterine bleeding

Table III. Different post-operative morbidities observed in relation to antibiotics used

Antibiotic

Prophylaxis

$\mathrm{n}(\%)$
Post-operative Morbidities

\begin{tabular}{rrrrr}
\hline Fever & UTI & $\begin{array}{r}\text { Wound } \\
\text { infection }\end{array}$ & $\begin{array}{r}\text { Vaginal } \\
\text { discharge }\end{array}$ & URTI \\
\hline $3(4.1)$ & $5(6.8)$ & $6(8.2)$ & $7(9.5)$ & $3(4.1)$ \\
$1(2)$. & $1(2.7)$ & 0 & 0 & $2(5.5)$ \\
\hline
\end{tabular}

$\mathrm{UTI}=$ Urinary tract infection

URTI = Upper respiratory tract infection 


\section{Table IV. Post-operative morbidity after Ampicillin prophylaxis}

\begin{tabular}{lrr} 
Ampicillin & \multicolumn{2}{c}{ Post-operative morbidity* } \\
\cline { 2 - 3 } prophylaxis & $\begin{array}{r}\text { Absent } \\
(\mathrm{N}=81)\end{array}$ & $\begin{array}{r}\text { Present } \\
(\mathrm{N}=28) \\
\mathrm{n}(\%)\end{array}$ \\
& $49(60.4)$ & $24(85.7)$ \\
\hline Given $(\mathrm{n}=73)$ & $32(39.5)$ & $4(14.2)$ \\
\hline Not given $(\mathrm{n}=36)$ &
\end{tabular}

*p-value: 0.014

prophylaxis was used than in those patients who were not receiving ampicillin as prophylaxis (table IV).

\section{Discussion}

The most interesting finding of our study is that the post-operative complications were less common in patients with pathogenic bacteria on pre-operative vaginal swab culture than in those patients where no pathogens were isolated. The culture positive patients received antibiotics according to the respective sensitivity of antibiotics. These antibiotics were given either alone or in combination therapy.

The findings explain important concerns. First, our study confirms the beneficial effect of pre-operative vaginal swab culture in elective gynaecological surgery (table II) especially in uterine prolapse patients. Studies have emphasized that within colonised tissues, such as the female genital tract, organisms that are relatively dominant in numbers constitute a pathogen which can be cultured even from asymptomatic patients, ${ }^{7}$ whereas those species that are fewer in number may not be identified during primary isolation. Several studies confirm that the density of microbial colonisation appears to be relevant not only to the condition of asymptomatic individuals but also to the initiation of disease state. ${ }^{12,13,14}$

Secondly, our study showed that a sensitive and combination antimicrobial coverage is more effective in reducing post-operative morbidities for any patient undergoing elective surgery regardless of the presence or absence of specific risk factors. In one study it was shown that a broader combination of cefazolin and metronidazole provided better efficacy with regards to post-operative morbidity when compared with cefazolin alone. ${ }^{15}$ Various studies suggest that prophylaxis for hysterectomy should consist of an agent, or combination of agents, with activity against both aerobic and anaerobic bacteria. ${ }^{16}$ Some researchers hold the view that selective prophylaxis should be practiced instead of mass prophylaxis at least in elective gynaecological surgery as the most effective regimen of prophylaxis has yet not been established. ${ }^{17}$

Our study showed that the peri-operative ampicillin prophylaxis (administered in patients with nonpathogen on vaginal swab culture) is not sufficient to reduce post-operative complications in our setting. This could be due to ampicillin being active only against aerobic microbes, has limited spectrum thus does not meet the guidelines as an ideal agent for prophylaxis. The peri-operative antibacterial prophylaxis is effective in reducing post-operative infections only when apart from proper timing and duration, the appropriate selection of antimicrobial agents is taken into consideration. Although the majority $(86 \%)$ of patients received antibiotic prophylaxis prior to surgery, surveys have shown that optimal practice is not achieved in many hospital. ${ }^{18,19,20,21}$ These results highlight the challenges of disseminating evidence based protocols systematically into routine clinical practice. Our study supports previous reports that Gram-negative bacilli (E. coli, Klebsiella pneumoniae, and Proteus species) are part of the endogenous vaginal flora. ${ }^{4,22}$ We isolated $S$. aureus (19.6\%), and $E$. coli (11.9\%), Pseudomonas, Klebsiella, Enterococcus and streptococci from asymptomatic pre-operative vaginal swab culture. The principal microorganisms that cause wound infections after gynaecologic surgery are staphylococci, streptococci and Gram-negative bacilli. Reports have shown that the Gram-negative bacilli are introduced into the upper genital tract during instrumentation and/or gynaecologic surgery, ${ }^{6}$ while staphylococci and streptococci are inoculated into the wound from the skin, and the latter organisms are transferred from the pelvic cavity as the surgeon closes the abdominal wound. ${ }^{23}$

Our study design has some limitations such as the lack of microbiological data from women following surgery. This knowledge would have allowed us a better understanding of the role of the pathogen 
(and the prescribed antimicrobial prophylaxis regimen) in hysterectomy. For example, presence of a microorganism in the genital tract besides infection can also be found in instances of colonisation, creating difficulty in clarifying the exact pathogenic role in instances of infection.

Despite limitations our study identifies issues of concern in developing countries. As in India and many other developing countries, routine antimicrobial sensitivity testing is not available in all the hospitals, the peri-operative prophylaxis is based on surveys of antimicrobial susceptibility test. The microbes, however, are known to vary in strains, and in the resistant patterns in post-operative infections according to geographical locations. The decision to use a particular antimicrobial agent in a setting requires access to the guidelines and a comprehensive evaluation of the patient's specific circumstances. Different studies and guidelines of prophylaxis are generally from high-income countries and of limited value to guide in developing countries. ${ }^{5}$ This limitation may explain the difficulties in following specific guidelines in clinical practice. We believe that the traditional surgical classification schedule should also account for patient-specific risk factors. The development of local evidence based guidelines, and an outcome based document of appropriate antibiotic may help clinicians achieve a higher certainty to improve post-operative outcomes. This approach would reduce the risk of emerging resistant bacteria, super-infection, risks of toxic reactions and cost of therapy.

In conclusion, irrational antibiotic prophylaxis leads to excessive surgical wound infection. A common error in antibiotic prophylaxis is the selection of the incorrect antibiotic. Development of a local guideline based on the knowledge of the local bacterial aetiology and susceptibility patterns may provide an updated recommendation for optimal prophylaxis. Local issues should be considered when establishing recommendations for antimicrobial selection for gynaecological surgery.

\section{Acknowledgment}

The authors thank Dr VK Mahadik, Director, RD Gardi Medical College, for his constant support during the entire work. They also thank Dr Yogyata Marothi and Dr Rama lyer for microbiological assistance and the laboratory staff for their support in data collection.

\section{References}

1. Barclay L, Vega CP. Guidelines Issued on Antibiotic Prophylaxis for Gynecologic Procedure. Obstet Gynecol 2009; 113: 11801189.

2. Delgado-Rodriguez $M$, Sillero-Arenas $M$, Medina-Cuadros M, Martinez-Gallego G. Nosocomial infections in surgical patients: comparison of two measures of intrinsic patient risk. Infect Control Hosp Epidemiol 1997; 18: 19-23. http://dx.doi. org/10.1086/647495

3. Hemsell DL. Infections after gynecologic surgery. Obstet Gynecol Clin North Am 1989; 16: 381-400.

4. Rein MF. Vulvovaginitis and cervicitis. In Mandell GL, Bennett JE, Dolin R, eds. Principles and practice of infectious diseases. 4th ed. New York: Churchill Livingstone; 1995: 1070-1075.

5. Lestaries ES, Sererin JA, Filins PMG, et al. Antimicrobial resistance Indonesia. Prevalence and prevention (AMRID) Antimicrobial resistance among commensal isolates of Escherichia Coli and Staphylococcus aureus in the Indonesian Population inside and outside hospitals. Eur J. Clin, Microbiol Infect Dis 2008; 27(1): 45-51. http://dx.doi.org/10.1007/ s10096-007-0396-z

6. Duff P. The pathophysiology and management of postcesarean endomyometritis. Obstet Gynecol 1986; 67: 269-274. http:// dx.doi.org/10.1097/00006250-198602000-00021

7. Larsen B, Monif GRG. Understanding the bacterial flora of the female genital tract. Clin Infect Dis 2001; 32(4): e69-e77. http://dx.doi.org/10.1086/318710

8. Smaill F, Hofmeyr GJ. Antibiotic prophylaxis for cesarean section. Cochrane Database Syst Rev 2002, CD000933.

9. Löfgren M, Poromaa IS, Stjerndahl JH, Renström B. Postoperative infections and antibiotic prophylaxis for hysterectomy in Sweden: a study by the Swedish National Register for Gynecologic Surgery. Acta Obstet Gynecol Scand 2004; 83(12): 1202-1207.

10. Ledger WJ, Gee C, Lewis WP. Guidelines for antibiotic prophylaxis in gynecology. Am / Obstet Gynecol 1975; 121(8): 1038-1045.

11. Dettenkofer M, Forster DH, Ebner W, Gastmeier P, Ruden $\mathrm{H}$, Daschner FD. The practice of perioperative antibiotic prophylaxis in eight German hospitals. Infection 2002; 30: 164-167. http://dx.doi.org/10.1007/s15010-002-2170-6

12. Bartlett JG, Onderdonk AB, Drude E, et al. Quantitative bacteriology of the vaginal flora. J Infect Dis 1977; 136: 271277. http://dx.doi.org/10.1093/infdis/136.2.271

13. Roy S, Sharma M, Ayyagari A, Malhotra S. A quantitative study of bacterial vaginosis. Indian J Med Res 1994; 100: 172-176.

14. Monif GRG. Semiquantitative bacterial observations with group B streptococcal vulvovaginitis. Infect Dis Obstet Gynecol 1999; 7: 227-229.

15. Meyer NL, Hosier KV, Scott K, Lipscomb GH. Cefazolin versus cefazolin plus metronidazole for antibiotic prophylaxis at cesarean section. Southern medical journal 2003; 96: 992-995. http://dx.doi.org/10.1097/01.SMJ.0000060570.51934.14

16. Brown EM, Depares J, Robertson AA, Jones S, et al. Amoxycillinclavulanic acid (Augmentin) versus metronidazole as prophylaxis in hysterectomy: a prospective, randomized clinical trial. Br J Obstet Gynaecol 1988; 95(3): 286-293. http://dx.doi.org/10.1111/j.1471-0528.1988.tb06871.x

17. Houang ET. Antibiotic prophylaxis in hysterectomy and induced abortion. A review of the evidence. Drugs 1991; 41(1): 19-37. 
http://dx.doi.org/10.2165/00003495-199141010-00003

18. Dellinger EP, Hausmann S, Bratzler D et al. Hospitals collaborate to decrease surgical site infections. Am / Surg 2005; 190: 9-15. http://dx.doi.org/10.1016/j.amjsurg.2004.12.001

19. Yalcin AN, Serin S, Gurses E, Zencir M. Surgical antibiotic prophylaxis in Turkish University Hospital. J Chemother 2002; 14: 373-377.

20. Finkelstein R, Reinhertz G, Embom A. Surveillance of use of antibiotic prophylaxis in surgery. Isr / Med Science 1996; 32: 1093-1097.
21. Tourmousoglou CE, Yiannakopoulou ECh, Kalapothaki V, Bramis J, St Papadopoulos J. Adherence to guidelines for antibiotic prophylaxis in general surgery: a critical appraisal. J Antimicrob Chemother 2008; 61(1): 214-218. http://dx.doi. org/10.1093/jac/dkm406

22. Hemsell DL. Infections after gynecologic surgery. Obstet Gynecol Clin North Am 1989; 16: 381-400.

23. Gibbs RS, Blanco JD, St Clair PJ. A case-control study of wound abscess after cesarean delivery. Obstet Gynecol 1983; 62: 498-503 